\title{
First-principles approach with a pseudohybrid density functional for extended Hubbard interactions
}

\author{
Sang-Hoon Lee* and Young-Woo Son ${ }^{\dagger}$ \\ Korea Institute for Advanced Study, Seoul 02455, Korea
}

(Received 14 November 2019; revised 13 October 2020; accepted 7 December 2020; published 23 December 2020)

\begin{abstract}
For massive database-driven materials research, there are increasing demands for both fast and accurate quantum mechanical computational tools. Contemporary density functional theory (DFT) methods can be fast, sacrificing their accuracy, or be precise, consuming a significant amount of resources. Here, to overcome such a problem, we present a DFT method that exploits self-consistent determinations of the on-site and intersite Hubbard interactions $(U$ and $V$ ) simultaneously and obtain band gaps of diverse materials in the accuracy of the $G W$ method at a standard DFT computational cost. To achieve the self-consistent evaluation of $U$ and $V$, we adapt a recently proposed Agapito-Curtarolo-Buongiorno Nardelli pseudohybrid functional for $U$ to implement a density functional of $V$. This method is found to be appropriate for considering various interactions such as local Coulomb repulsion, covalent hybridization, and their coexistence. We also obtain good agreements between computed and measured band gaps of low-dimensional systems, thus meriting this approach for large-scale as well as high-throughput calculations for various bulk and nanoscale materials with a higher accuracy.
\end{abstract}

DOI: 10.1103/PhysRevResearch.2.043410

\section{INTRODUCTION}

Theoretical and computational methods based on the density functional theory (DFT) [1,2] have been indispensable tools in understanding the physical properties of real materials [3]. Although they fail quantitatively and sometimes qualitatively in calculating band gaps [3] with the local density approximation (LDA) [2] or the generalizedgradient approximation (GGA) [4], they are the only currently available methods without significant computational costs to offer fully quantum mechanical computational results for diverse phenomena involved with many thousands of electrons $[3,5]$. Thus, regardless of such shortcomings, DFTbased approaches prevail in data-driven materials research [6] spanning various areas such as energy materials [7-9], electronic applications [10-12], low-dimensional crystals [13], and topological materials [14-17]. These databases with improved accuracy will be of great benefit in advancing future technology.

To build high-quality materials databases, it is vital to improve the accuracy of DFT-based methods. Several methods beyond LDA and GGA have been suggested so far. The local Coulomb repulsion $U$ was introduced in DFT $+U$ to compensate the overdelocalization of $d$ or $f$ electrons in LDA or GGA $[18,19]$. Beyond the static correlation effectively treated in DFT $+U$, DFT with the dynamical mean-field theory [20-22]

\footnotetext{
*1shgoal@kias.re.kr

†hand@kias.re.kr

Published by the American Physical Society under the terms of the Creative Commons Attribution 4.0 International license. Further distribution of this work must maintain attribution to the author $(s)$ and the published article's title, journal citation, and DOI.
}

has been used for strongly interacting materials. The quasiparticle energy of semiconductors can be obtained accurately with the $G W$ approximations [23-25]. Hybrid functionals such as Heyd-Scuseria-Ernzerhof (HSE) [26,27] and LDA with the modified Becke-Johnson exchange potential (mBJLDA) [28] are also popular. However, all the methods above except DFT $+U$ and mBJLDA involve intensive computations, discouraging their use in data-driven research. Due to some limitations in the latter [29], we will focus on improving the former for high-throughput calculations.

Two aspects in the DFT $+U$ formalism are important in obtaining accurate band gaps $a b$ initio. First, the onsite Hubbard $U$ needs to be estimated self-consistently [30] and various methods for this purpose [30-37] have been suggested. Among them, the direct evaluation from the Hartree-Fock (HF) formalism [35-37] is relevant here since other methods involve additional expensive calculations. A recent proposal by Agapito-Curtarolo-Buongiorno Nardelli (ACBN0) [37] allows a direct self-consistent evaluation of $U$. They demonstrated improved agreements with experiments with a negligible increase in computational cost [37,38]. Second, the intersite Hubbard $V$ between the localized orbital of interest in DFT $+U$ and its neighboring orbitals also needs to be considered properly because it could lead to better descriptions of the electronic structures of some solids [39-41]. Moreover, DFT $+U$ hardly improves the LDA and GGA gaps of simple semiconductors such as $\mathrm{Si}$ while $\mathrm{DFT}+U$ with $V$ does [40]. Therefore, by combining these advancements, we may obtain an efficient large-scale and high-throughput computational tool for materials research.

In this paper, we extend the ACBNO functional for $\mathrm{DFT}+U$ [37] to implement a density functional for the intersite Coulomb interaction of $V$. With this, we achieve excellent agreements between the self-consistent $a b$ initio band gaps of diverse semiconductors and insulators and those 
from experiments. Band gaps comparable to those from $G W$ methods $[24,25]$ can be obtained within the standard DFTGGA computational time. Moreover, for low-dimensional systems in which the screening of the Coulomb interaction varies significantly, this method can also compute accurate band gaps of few-layer black phosphorus and a $\mathrm{Si}(111)$ $(2 \times 1)$ surface, respectively, demonstrating its flexibility with structural and dimensional variations. Considering the recent explosive expansion of data-driven materials research using the DFT [6-17], the improved accuracy in DFT computations is of great importance in constructing useful and reliable databases of materials. Thus, we expect that this approach could accelerate efficient high-throughput calculations with better accuracy for materials discoveries.

This paper is organized as follows. We first introduce our formalism of the ACBN0-like functional for the intersite Hubbard interactions in Sec. II. Then, using the method described in Sec. III, we present our computational results of energy band gaps of various three-dimensional solids in Sec. IV and those of low-dimensional systems in Sec. V. Finally, we discuss several aspects of this functional and conclude in Sec. VI.

\section{FORMALISM}

Let us first consider the mean-field (MF) energy of the Coulomb interaction between electrons in a pair of atoms $I$ and $J$ with the HF approximation,

$$
\begin{aligned}
E_{\mathrm{MF}}= & \frac{1}{2} \sum_{I J} \sum_{i j} \sum_{\sigma \sigma^{\prime}}\left\langle\phi_{i}^{I} \phi_{j}^{J}\left|V_{e e}\right| \phi_{i}^{I} \phi_{j}^{J}\right\rangle \\
& \times\left(n_{i i}^{I I \sigma} n_{j j}^{J J \sigma^{\prime}}-\delta_{\sigma \sigma^{\prime}} n_{i j}^{I J \sigma} n_{j i}^{J I \sigma^{\prime}}\right) .
\end{aligned}
$$

In the abbreviated representation of pairwise HF energy in Eq. (1), the general occupation matrix is written as

$$
\begin{aligned}
n_{i j}^{I J \sigma} & \equiv n_{i j}^{I, n, l, J, n^{\prime}, l^{\prime}, \sigma} \\
& =\sum_{m \mathbf{k}} w_{\mathbf{k}} f_{m \mathbf{k}}\left\langle\psi_{m \mathbf{k}}^{\sigma} \mid \phi_{i}^{I, n, l}\right\rangle\left\langle\phi_{j}^{J, n^{\prime}, l^{\prime}} \mid \psi_{m \mathbf{k}}^{\sigma}\right\rangle,
\end{aligned}
$$

where $f_{m \mathbf{k}}$ is the Fermi-Dirac function of the Bloch state $\left|\psi_{m \mathbf{k}}^{\sigma}\right\rangle$ with a spin $\sigma$ of the $m$ th band at a momentum $\mathbf{k}$ and $w_{m \mathbf{k}}$ is the $\mathbf{k}$-grid weight. The Löwdin orthonormalized atomic wave function $\left(\left|\phi_{i}^{I, n, l}\right\rangle\right)$ is used as a projector for the localized atomic orbital having the principal, azimuthal, and angular quantum numbers of $n, l$, and $i$, respectively, at an atom $I$. We will use a brief notation for atom $I$ representing specific principal and azimuthal quantum numbers of $n$ and $l$ of the Ith atomic element in a solid hereafter. We also note that the diagonal terms in Eq. (2) are the usual on-site occupations for DFT $+U$. In Eq. (1), we neglect other small pairwise interactions, e.g., the cross-charge exchanges between neighbors [40], and discuss their effects in Appendix A and in Table V.

Assuming the effective interactions of $\left\langle V_{e e}\right\rangle$ in Eq. (1) are all equal to their atomic average [40], the rotationally invariant or angular momentum averaged form of $E_{\mathrm{MF}}$ can be written as $E_{\mathrm{MF}}=E_{\mathrm{Hub}}=E_{U}+E_{V}$, where $E_{U}$ is for the case of $I=J$ and $E_{V}$ for $I \neq J . E_{U}$ is the well-known energy functional for $U$ suggested by Dudarev et al. [42]. For the $I \neq J$ case where atoms $I$ and $J$ locate at different positions, respectively,

$$
E_{V}=\frac{1}{2} \sum_{\{I, J\}} \sum_{i j} \sum_{\sigma \sigma^{\prime}} V^{I J}\left(n_{i i}^{I I \sigma} n_{j j}^{J J \sigma^{\prime}}-\delta_{\sigma \sigma^{\prime}} n_{i j}^{I J \sigma} n_{j i}^{J I \sigma^{\prime}}\right),
$$

where the $\{I, J\}$ indicates the summation for pairs of atoms $I$ and $J$ of which the interatomic distance of $d_{I J}$ is less than a given cutoff. In Eq. (3), $V^{I J}$ is the intersite Hubbard interaction for the pair and will be determined based on the method of ACBNO [37].

To obtain a functional form of $V^{I J}$, as is discussed for $U$ in Ref. [37], we also follow a central ansatz by Mosey et al. $[35,36]$ that leads to a "renormalized" occupation number for the pair such as

$$
\begin{aligned}
N_{\psi_{m \mathbf{k}}}^{I J \sigma} \equiv & N_{\psi_{m \mathbf{k}}}^{I, n, l, n^{\prime}, l^{\prime} \sigma} \\
= & \sum_{\{I\}} \sum_{i}\left\langle\psi_{m \mathbf{k}}^{\sigma} \mid \phi_{i}^{I, n, l}\right\rangle\left\langle\phi_{i}^{I, n, l} \mid \psi_{m \mathbf{k}}^{\sigma}\right\rangle \\
& +\sum_{\{J\}} \sum_{j}\left\langle\psi_{m \mathbf{k}}^{\sigma} \mid \phi_{j}^{J, n^{\prime}, l^{\prime}}\right\rangle\left\langle\phi_{j}^{J, n^{\prime}, l^{\prime}} \mid \psi_{m \mathbf{k}}^{\sigma}\right\rangle,
\end{aligned}
$$

where the summation performs for all the orbitals of a type $I$ atom with quantum number $n$ and $l$ [denoted by $\{I\}$ in Eq. (4)] and for a type $J$ atom with $n^{\prime}$ and $l^{\prime}$ (denoted by $\{J\}$ ) in a given unit cell, respectively. Here, we note that the sum is only obtained for all the given pairs within a specific distance. Therefore, we can obtain the ACBNO-like functional for $V^{I J}$ that effectively accounts for the screening in the bond region between the pair, e.g., the interaction between the $l$ th orbital of the $I$ atom and the $l^{\prime}$ th orbital of the $J$ atom. Corresponding to the ACBN0 functional [37] for $U$, we can replace $n_{i j}^{I J \sigma}$ in Eq. (1) by a renormalized density matrix for the pair,

$$
\left.P_{i j}^{I J \sigma}=\sum_{m \mathbf{k}} w_{m \mathbf{k}} f_{n \mathbf{k}} N_{\psi_{m \mathbf{k}}}^{I J \sigma}\left|\psi_{m \mathbf{k}}^{\sigma}\right| \phi_{i}^{I}\right\rangle\left\langle\phi_{j}^{J} \mid \psi_{m \mathbf{k}}^{\sigma}\right\rangle .
$$

In the case where there are no electrons participating in the bond between atoms $I$ and $J$, the renormalized density matrix of Eq. (5) for the pair automatically reduces to zero, thereby nullifying the intersite effects. Note that $I$ and $J$ here also implicitly include orbital indices.

The bare Coulomb interaction between electrons belonging to the pair can be expressed by the electron repulsion integral [37],

$$
V_{\mathrm{ERI}} \equiv(i k \mid j l) \equiv \int d \mathbf{r}_{1} d \mathbf{r}_{2} \frac{\phi_{i}^{I *}\left(\mathbf{r}_{1}\right) \phi_{k}^{I}\left(\mathbf{r}_{1}\right) \phi_{j}^{J *}\left(\mathbf{r}_{2}\right) \phi_{l}^{J}\left(\mathbf{r}_{2}\right)}{\left|\mathbf{r}_{1}-\mathbf{r}_{2}\right|},
$$

where $i$ and $k$ are orbital indices belonging to atom $I$ and $j$ and $l$ to atom $J$. Using Eqs. (5) and (6), the ACBNO-like energy expression $\left(E_{\mathrm{ACBN} 0}^{V}\right)$ for the intersite Hubbard interaction can be written as

$$
\begin{aligned}
E_{\mathrm{ACBN} 0}^{V}= & \frac{1}{4} \sum_{\{I, J\}} \sum_{i j k l} \sum_{\sigma, \sigma^{\prime}}\left[P_{i k}^{I I \sigma} P_{j l}^{J J \sigma^{\prime}}-\delta_{\sigma \sigma^{\prime}} P_{i l}^{I J \sigma} P_{j k}^{J I \sigma^{\prime}}\right] \\
& \times(i k \mid j l),
\end{aligned}
$$

where the additional prefactor of $1 / 2$ arises from a double counting of the same pairs. Equating Eq. (3) to Eq. (7), we 
can then obtain a density functional form of $V^{I J}$,

$$
V^{I J}=\frac{1}{2} \frac{\sum_{\sigma, \sigma^{\prime}} \sum_{i j k l}\left[P_{i k}^{I I \sigma} P_{j l}^{J J \sigma^{\prime}}-\delta_{\sigma \sigma^{\prime}} P_{i l}^{I J \sigma} P_{j k}^{J I \sigma^{\prime}}\right](i k \mid j l)}{\sum_{\sigma, \sigma^{\prime}} \sum_{i j}\left[n_{i i}^{I I \sigma} n_{j j}^{J J \sigma^{\prime}}-\delta_{\sigma \sigma^{\prime}} n_{i j}^{I J \sigma} n_{j i}^{J I \sigma^{\prime}}\right]} .
$$

An energy functional for $V$ can be constructed by subtracting a double-counting term $\left(E_{V}^{\mathrm{dc}}\right)$ from $E_{V}$ in Eq. (3). Following the discussion in Ref. [40], we use the fully localized limit and then $E_{V}^{\mathrm{dc}}=\sum_{\{I, J\}} \sum_{i, j} \sum_{\sigma, \sigma^{\prime}} \frac{V^{I J}}{2} n_{i i}^{I I \sigma} n_{j j}^{J J \sigma^{\prime}}$. With this, the final functional for the intersite interaction of $V$ can be written as

$$
E_{V}[\{\mathbf{n}\}]=-\frac{1}{2} \sum_{\{I, J\}} \sum_{\sigma} V^{I J}[\{\mathbf{n}\}] \operatorname{Tr}\left[\mathbf{n}^{I J \sigma} \mathbf{n}^{J I \sigma}\right],
$$

where $\mathbf{n}^{I J \sigma}$ is the matrix notation for the general occupation in Eq. (2), $\{\mathbf{n}\}=\left\{\mathbf{n}^{I I \sigma}, \mathbf{n}^{I J \sigma}\right\}$ and $V^{I J}[\{\mathbf{n}\}]$ in Eq. (8). For the on-site repulsion, we used the ACBN0 functionals in Eqs. (12) and (13) of Ref. [37] so that we complete a construction of the pseudohybrid-type functionals for the two essential Hubbard interactions. As discussed before [40], the minus sign in $E_{V}[\{\mathbf{n}\}]$ highlights the role of the intersite Hubbard interaction that localizes electrons between atoms $I$ and $J$. So, Eq. (9) implemented in this study can improve the description of covalent bonding or augment the overlocalization [41] caused by $U$ in the case where the bonding between neighboring $d$ and $p$ orbitals plays important roles for the various ground-state properties of solids.

\section{COMPUTATIONAL METHODS}

We implemented $E_{V}[\{\mathbf{n}\}]$ in Eq. (9), ACBN0 functionals, and other related quantities in the QUANTUM ESPRESSO package [43]. For the Kohn-Sham potential corresponding to Eq. (9), we used Eq. (13) in Ref. [40]. To compute $V_{\text {ERI }}$ in Eq. (6), we used the pseudo-atomic orbitals (PAO) expressed as a linear combination of Gaussian-type functions (PAO-3G) for the basis set as in Ref. [37]. With the aid of the PyQuante package [44], the integrals were done quickly in an analytical way. For all calculations here, the cutoff for $d_{I J}$ sets within the second-nearest neighbors. We will discuss the effects of $d_{I J}$ later. The on-site interactions for $s$ orbitals were neglected for all materials considered here while for intersite interactions, $s$ orbitals were included. Fully converged $U$ and $V$ were obtained when the difference in energy between two consecutive self-consistent steps is less than $10^{-8} \mathrm{Ry}$. We used the GarrityBennett-Rabe-Vanderbilt (GBRV) ultrasoft pseudopotentials [45]. Regarding the pseudopotential dependence of ACBN0 functionals [38], we tested the norm-conserving pseudopotentials provided by the PseudoDoJo project [46] and will discuss its effects in Appendix B. The kinetic energy cutoff was set to $160 \mathrm{Ry}$ to fix the value for all materials. The Brillouin zone (BZ) integration was performed with a $\Gamma$-centered $\mathbf{k}$-point grid spacing of $0.2 \AA^{-1}$. The lattice structures are chosen from the experimentally available data for comparison to the results with other computational methods and otherwise are relaxed within a standard DFT scheme. For low-dimensional materials discussed in Sec. V, we used slightly modified setups for com-
TABLE I. Calculated band gaps (in eV). For comparison, gaps from experiments and other methods are also shown. Structures denoted by the strukturbericht designation are in parentheses, except monoclinic $\mathrm{ZrO}_{2}$. Experimental data are from Refs. [28,29,37,47]

\begin{tabular}{|c|c|c|c|c|c|c|}
\hline Solid & $\mathrm{GGA}^{\mathrm{a}}$ & ACBNO & This work & $\mathrm{HSE}^{\mathrm{b}}$ & $G W^{\mathrm{c}}$ & Expt. \\
\hline C (A4) & 4.15 & 4.17 & 5.50 & 5.43 & 6.18 & 5.50 \\
\hline $\mathrm{Si}$ (A4) & 0.58 & 0.52 & 1.36 & 1.21 & 1.41 & 1.17 \\
\hline Ge (A4) & 0.00 & 0.00 & 0.61 & 0.80 & 0.95 & 0.74 \\
\hline BP (B3) & 1.25 & 1.24 & 2.27 & 2.13 & 2.20 & 2.40 \\
\hline AlP (B3) & 1.59 & 2.00 & 2.66 & 2.42 & 2.90 & 2.50 \\
\hline GaP (B3) & 1.60 & 1.74 & 2.47 & 2.39 & 2.80 & 2.35 \\
\hline InP (B3) & 0.67 & 0.94 & 1.46 & 1.77 & 1.44 & 1.42 \\
\hline AlAs (B3) & 1.43 & 1.75 & 2.43 & 2.13 & 2.18 & 2.23 \\
\hline GaAs (B3) & 0.55 & 0.68 & 1.28 & 1.11 & 1.85 & 1.52 \\
\hline InAs (B3) & 0.00 & 0.00 & 0.46 & 0.57 & 0.31 & 0.42 \\
\hline $\mathrm{SiC}$ (B3) & 1.37 & 1.74 & 2.49 & 2.32 & 2.88 & 2.42 \\
\hline BN (B3) & 4.48 & 5.14 & 6.31 & 5.91 & 7.14 & 6.36 \\
\hline ZnS (B3) & 2.09 & 3.43 & 3.71 & 3.44 & 4.15 & 3.80 \\
\hline $\mathrm{ZnSe}$ (B3) & 1.28 & 2.32 & 2.60 & 2.38 & 2.66 & 2.82 \\
\hline $\mathrm{ZnTe}$ (B3) & 1.31 & 1.99 & 2.30 & 2.34 & 2.15 & 2.39 \\
\hline $\mathrm{LiF}(\mathrm{B} 1)$ & 9.12 & 13.74 & 14.26 & 13.28 & 15.90 & 14.20 \\
\hline $\mathrm{MgO}$ (B1) & 4.80 & 8.84 & 10.06 & 6.59 & 9.16 & 7.90 \\
\hline $\mathrm{ZrO}_{2}{ }^{\mathrm{d}}$ & 3.74 & 5.10 & 5.97 & 5.20 & 5.34 & 5.50 \\
\hline $\mathrm{TiO}_{2}(\mathrm{C} 4)$ & 1.89 & 3.02 & 4.18 & 3.25 & 4.48 & 3.30 \\
\hline $\mathrm{MnO}(\mathrm{B} 1)$ & 0.91 & 2.56 & 2.73 & 4.77 & 3.50 & 3.60 \\
\hline $\mathrm{NiO}$ (B1) & 0.96 & 3.70 & 3.90 & 4.09 & 4.80 & 4.30 \\
\hline $\mathrm{ZnO}(\mathrm{B} 4)$ & 0.89 & 3.62 & 3.88 & 2.11 & 3.80 & 3.44 \\
\hline $\mathrm{Cu}_{2} \mathrm{O}(\mathrm{C} 3)$ & 0.55 & 1.28 & 1.52 & 2.02 & 1.97 & 2.17 \\
\hline MARE (\%) & 52.71 & 30.26 & 10.64 & 11.83 & 13.62 & \\
\hline MRE (\%) & -52.71 & -28.78 & 0.47 & -2.61 & 7.76 & \\
\hline
\end{tabular}
and references therein.

${ }^{\mathrm{a} G G A}$ by Perdew-Burke-Ernzerhof (PBE) [4].

${ }^{\mathrm{b}}$ All data from HSE06 results in Ref. [48] except $\mathrm{ZrO}_{2}$ from HSE06 in Ref. [49], $\mathrm{TiO}_{2}$ from HSE03 in Ref. [50], $\mathrm{ZnO}$ from HSE03 in Ref. [51], and $\mathrm{Cu}_{2} \mathrm{O}$ from HSE06 in Ref. [52].

${ }^{\mathrm{c}}$ All data from self-consistent $G W$ ( $\mathrm{sc} G W$ ) calculation results in Ref. [25] except GaP, InP, AlAs from scGW in Ref. [53], ZnSe from a partially self-consistent $G W\left(G W_{0}\right)$ in Ref. [54], BP from $G W_{0}$ in Ref. [55], $\mathrm{ZrO}_{2}$ from $\mathrm{sc} G W$ in Ref. [56], $\mathrm{TiO}_{2}$ from $\mathrm{sc} G W$ in Ref. [57], and $\mathrm{Cu}_{2} \mathrm{O}$ from $\mathrm{sc} G W$ in Ref. [58].

${ }^{\mathrm{d}}$ Monoclinic structure.

putations and presented detailed methods in the corresponding sections.

\section{ENERGY GAPS OF THREE-DIMENSIONAL SOLIDS}

We first tested our method for selected bulk solids with diverse characteristics. Table I and Fig. 1 summarize the calculated band gaps of 23 solids. We also listed the results from other calculations and measurements. We select solids from group IV, group III-V semiconductors, ionic insulators, metal chalcogenides, and metal oxides. Our calculated band gaps are in excellent agreement with experiments and are as accurate as those from the HSE and $G W$ methods as shown in Table I. The mean absolute relative error (MARE) with respect to the experimental data indicates that our method, and the HSE and $G W$ methods are closer to experiments than Perdew-Burke-Ernzerhof (PBE) and ACBNO. 


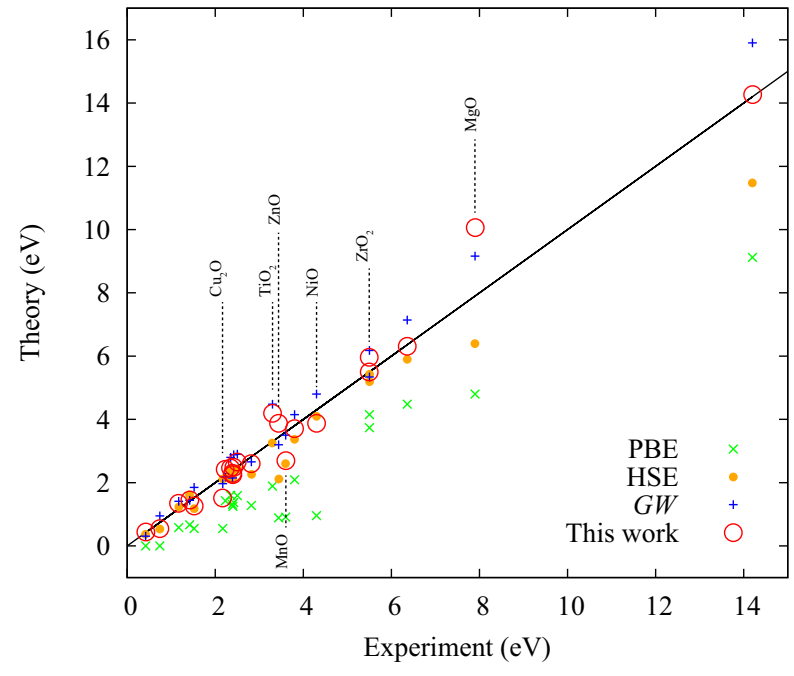

FIG. 1. Experimental vs theoretical band gaps in Table I. Metal oxides are marked and all other materials considered here are almost right on top of the experimental values.

The mean relative error (MRE) shows that PBE and ACBN0 underestimate the gaps (minus sign) while the $G W$ method overestimates them. Hereafter, we mainly focus on the calculated gap values and, for future reference, the band structures of all solids are displayed in the Supplemental Material (SM) [59].

For the group IV semiconductors, the effect of $U$ on the band gaps is almost negligible as shown in Table I (see the ACBN0 column) while the intersite Hubbard terms improve the band gaps dramatically, as was also discussed in a previous study using the linear-response theory [40].

For the group III-V semiconductors, both $U$ and $V$ affect their electronic structures because of their mixed covalent and ionic bonding characters. Therefore, ACBNO improves the PBE gaps and the intersite terms increase these further to match the experimental values. Details of computations such as self-consistent $U$ and $V$ for $\mathrm{Si}$ and GaAs compared with Ref. [40] are discussed below. We note that PBE and ACBN0 incorrectly describe Ge and InAs as a metal and a topological insulator, respectively, while our method confirms them as semiconductors such as HSE and $G W$ results.

We present the calculated $U$ and $V$ values for $\mathrm{Si}$ and $\mathrm{GaAs}$ to compare with the values obtained by the linear-response approach [40]. Table II shows the calculated results. Our onsite $U$ value for the $p$ orbital of $\mathrm{Si}$ is larger than that reported in a previous study [40]. All the other intersite terms except one between $p$ orbitals are smaller than those from the linearresponse approach [40]. We note here that the on-site term for Si has no effect on the band gap at all. Despite the differences in the Hubbard parameters, the calculated band gap of Si is in good agreement with the previous linear-response theory work [40] and experimental value. In the case of GaAs, our on-site interactions for the $p$ orbitals of Ga and As, respectively, are all smaller than the previous results [40]. As the $\mathrm{Si}$ case, our intersite values are also smaller than the previous results except for the one between the $p$ orbitals. Nevertheless, our computed band gap of $1.28 \mathrm{eV}$ for $\mathrm{GaAs}$ is larger than
TABLE II. Calculated $U$ and $V$ between $s$ and $p$ orbitals of the first nearest neighbors of $\mathrm{Si}$ and $\mathrm{GaAs}$ (in eV). Here, we compare our results with those based on the linear-response theory (LRT). For GaAs, the first (second) $U_{p}$ for on-site Hubbard interactions on the Ga (As) $p$ orbital. $V_{s p}\left(V_{p s}\right)$ corresponds to intersite terms between the Ga $s(p)$ orbital and As $p(s)$ orbital. $V_{p p}$ denotes the intersite interaction between the Ga $p$ orbital and As $p$ orbital.

\begin{tabular}{lcccccc}
\hline \hline & & $U_{p}$ & $V_{s s}$ & $V_{s p}$ & $V_{p s}$ & $V_{p p}$ \\
\hline Si & This work & 3.50 & 0.90 & 0.72 & 0.72 & 1.85 \\
& LRT $^{\mathrm{a}}$ & 2.82 & 1.40 & 1.36 & 1.36 & 1.34 \\
\multirow{2}{*}{ GaAs } & This work & $0.37,1.88$ & 0.91 & 1.26 & 0.80 & 1.75 \\
& LRT $^{\mathrm{a}}$ & $3.14,4.24$ & 1.75 & 1.76 & 1.68 & 1.72 \\
\hline \hline
\end{tabular}

${ }^{a}$ Reference [40].

the value of $0.90 \mathrm{eV}$ reported in Ref. [40] and is close to the experimental gap of $1.52 \mathrm{eV}$ (see Table I).

In the case of the ionic compound $\mathrm{LiF}$, the on-site $U$ improves the PBE band gap significantly because of its strong local Coulomb repulsion. Nonetheless, the intersite $V$ still increases the ACBN0 gap further to match with an experimental value.

A similar trend is also found in metal monochalcogenides (here $\mathrm{Zn}$ compounds only). For these compounds, the $U$ and $V$ functionals play similar roles as they do for $\mathrm{LiF}$ so that our results with $U$ and $V$ are quite closer to experimental values than those with $U$ only. We note that the calculated gaps depend on the choice of the pseudopotential of $\mathrm{Zn}$ while there is no such dependence in the cases of IV and III-V semiconductors. We will discuss this further for cases of metal oxides below.

Regarding metal oxides, our results agree with the calculations by other advanced methods. For $\mathrm{TiO}_{2}, \mathrm{MnO}, \mathrm{NiO}$, and $\mathrm{ZnO}$ in Table I, our ACBNO results already improve the PBE gaps significantly, similar with previous studies $[37,38,60]$ that calculated the detailed electronic structures with ACBNO. Our gaps are slightly larger than the values in other works $[37,38,60]$. These discrepancies originate from the different self-consistent $U$ values. With the intersite $V$ included, the changes in the on-site $U$ lead to an increase in the ACBNO gaps of $\mathrm{TiO}_{2}, \mathrm{MnO}, \mathrm{ZnO}$, and $\mathrm{NiO}$ as shown in Table III. We note that the gaps of the metal oxides depend on the choice of pseudopotentials. With the potentials from the PseudoDoJo project [46], we achieve a better agreement (Table VI in Appendix B). Because the effects of on-site and intersite interactions depend on the degree of localization or cutoff in the projector for the localized orbital [38], it is important to select or generate pseudopotentials with care to obtain accurate results [61] or to develop a computational method for the on- and intersite Hubbard interactions that do not depend on projectors.

For $\mathrm{Cu}_{2} \mathrm{O}$ and $\mathrm{Zr}_{2} \mathrm{O}$, our results are comparable to those from HSE and $G W$ calculations. We note here that for $\mathrm{Cu}_{2} \mathrm{O}$ the calculated energetic position of fully filled $d$ orbitals is lower than that of the HSE value [52] by $\sim 0.9 \mathrm{eV}$ (Fig. S7 in $\mathrm{SM})$. Since the degree of screening depends on the occupancy of orbitals in the ACBNO formalism, the weak screening for the fully filled $d$ orbitals such as cuprite seems to be 
TABLE III. Self-consistent on-site energies of $3 d$ orbitals of the transition metal $\left(U_{d}\right)$ and $2 p$ orbitals of oxygen $\left(U_{p}\right)$ and direct band gaps $\left(E_{g}\right)$ of selected transition metal oxides within the ACBN0 formalism (in eV). We also list the self-consistent on-site and intersite Hubbard interactions $\left(V_{d p}\right)$ between the metal $d$ and oxide $p$ orbitals, and $E_{g}$ using the current method.

\begin{tabular}{lccccc}
\hline \hline Solids & & $U_{d}$ & $U_{p}$ & $V_{d p}$ & $E_{g}$ \\
\hline $\mathrm{TiO}_{2}$ & This work $^{\mathrm{a}}$ & 0.27 & 8.49 & & 3.02 \\
& ACBNO $^{\mathrm{b}}$ & 0.15 & 7.34 & & 2.83 \\
& ACBNO $^{\mathrm{c}}$ & 0.96 & 10.18 & & 3.21 \\
& This work $^{\mathrm{d}}$ & 0.37 & 8.21 & 2.94 & 4.18 \\
$\mathrm{MnO}$ & This work $^{\mathrm{a}}$ & 5.11 & 2.99 & & 3.05 \\
& ACBN0 $^{\mathrm{b}}$ & 4.67 & 2.68 & & 2.83 \\
& ACBNo $^{\mathrm{c}}$ & 4.68 & 5.18 & & 2.65 \\
& This work $^{\mathrm{d}}$ & 5.31 & 2.94 & 2.72 & 3.60 \\
$\mathrm{NiO}$ & This work $^{\mathrm{a}}$ & 8.22 & 2.83 & & 4.66 \\
& ACBN0 $^{\mathrm{b}}$ & 7.63 & 3.00 & & 4.29 \\
& ACBN0 $^{\mathrm{c}}$ & 6.93 & 2.68 & & 4.14 \\
& This work $^{\mathrm{d}}$ & 7.77 & 2.37 & 2.93 & 5.13 \\
\multirow{2}{*}{$\mathrm{ZnO}$} & This work $^{\mathrm{a}}$ & 15.06 & 7.30 & & 3.62 \\
& ACBN0 $^{\mathrm{b}}$ & 12.80 & 5.29 & & 2.91 \\
& ACBN0 $^{\mathrm{c}}$ & 13.30 & 5.95 & & 3.04 \\
& This work $^{\mathrm{d}}$ & 14.96 & 7.07 & 3.01 & 3.88 \\
& & & & & \\
\hline \hline
\end{tabular}

${ }^{\mathrm{a}} \mathrm{DFT}+U$.

${ }^{\mathrm{b}}$ Reference [37].

${ }^{\mathrm{c}}$ Reference [38].

${ }^{\mathrm{d}} \mathrm{DFT}+U+V$.

inevitable. Thus, it needs to improve the way it treats the completely filled $d$ orbitals with $U$ and $V$ within this formalism. Regardless of its limitation, we found that our computed gaps with $V$ are considerably improved if compared with those from ACBN0 and mBJLDA. Considering limits in mBJLDA to obtain gaps for these compounds [29], our method could be a good alternative tool for studying zirconia and cuprite.

To compare with the previous ACBN0 studies on metal oxides [37,38], we consider the on-site Hubbard interaction of $U_{d}$ for the $d$ electrons of metals and $U_{p}$ for the $p$ electrons of oxygen in $\mathrm{TiO}_{2}, \mathrm{MnO}, \mathrm{NiO}$, and $\mathrm{ZnO}$, respectively. We also provide those values and the first-nearest-neighbor intersite Hubbard interaction terms (the $d$ - $p$ interactions) calculated with our method. The results are summarized in Table III. We note that except for $\mathrm{TiO}_{2}$ our on-site repulsions for the $d$ orbitals of metals are rather larger than the values from previous works $[37,38]$ while the repulsions for oxide $p$ orbitals are similar with previous studies. Since the size of the on-site repulsion of the $d$ orbital is almost proportional to the size of the gap, our larger estimations of $U_{d}$ result in relatively larger direct energy gaps for $\mathrm{MnO}, \mathrm{NiO}$, and $\mathrm{ZnO}$, respectively, if compared with the previous studies $[37,38]$. By including the intersite $V_{d p}$, the gaps increase further because of a reduction in the energetic position of the conduction band maximum at the $\Gamma$ point (see Fig. S6 in SM). As mentioned in Ref. [38], the discrepancies of $U_{d}, U_{p}$, and $E_{g}$ may be attributed to the way the Coulomb integrals are calculated and the treatment of the localized orbitals. As shown in Table VI, our gap values also change according to the different choice of pseudopotentials. Therefore, a further study on this problem is required (a)

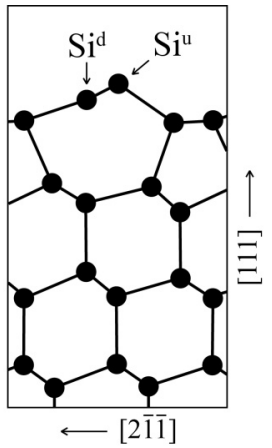

(b)

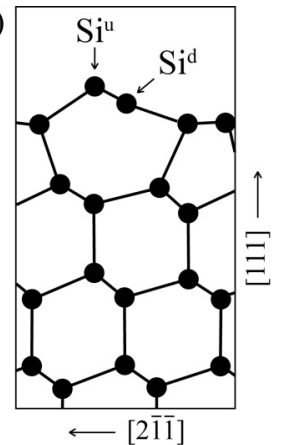

(c)

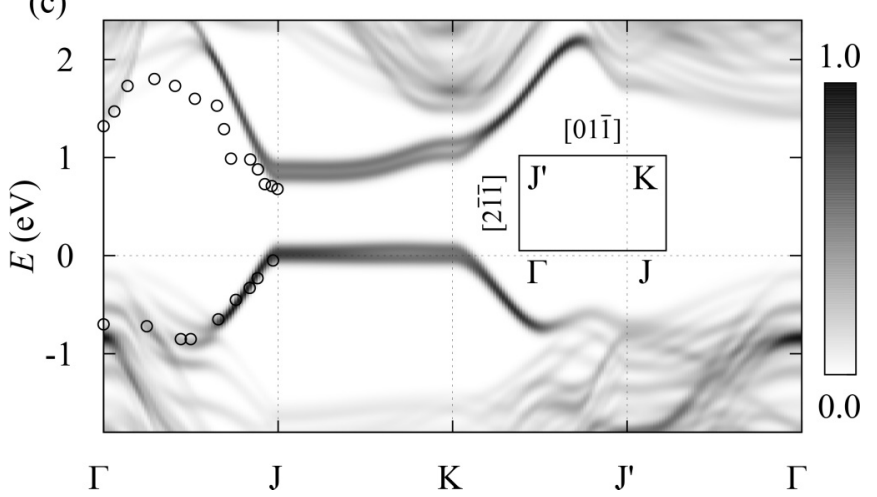

FIG. 2. The cross-sectional views of optimized atomic structures for two energetically degenerate buckled structures are shown in (a) and (b). Si atom (solid circles) relaxed down to (up away from) the surface is denoted by $\mathrm{Si}^{\mathrm{d}}$ and $\mathrm{Si}^{\mathrm{u}}$, respectively. (c) Averaged surface band structures projected to the first four layers of $\operatorname{Si}(111)-(2 \times 1)$ where the scale on the right-hand side denotes the local density of states in an arbitrary unit. The energetic position of the bulk valence band maximum at $\Gamma$ is set to zero. Black open circles are experimental data from direct [66] and inverse [67] photoemission spectroscopic experiments. The inset describes the BZ.

to obtain a better description of the electronic structures of metal oxides.

\section{ENERGY GAPS IN LOW-DIMENSIONAL MATERIALS}

Now, we consider low-dimensional systems where the screening of the Coulomb interaction varies rapidly. The $G W$ approximation calculates the quasiparticle gaps quite accurately but its convergence is very slow with respect to the k-point density and other parameters [62,63]. The hybrid functional methods do not suffer such an issue but they produce unreliable gap values with structural or dimensional variations [64]. The mBJLDA, another low-cost alternative for bulk solids, also suffers a similar problem as hybrid functionals [65]. However, with our method, self-consistently computed occupations of atoms at the boundary and bulk reflect the screening of the Coulomb interaction through Eqs. (4), (5), and (8). Hence, we expect that the current method may overcome the aforementioned difficulties for low-dimensional materials.

To test this method, we first calculated the electronic structures of a $\operatorname{Si}(111)-(2 \times 1)$ surface. Because of a unique surface reconstruction resulting in a quasi-one-dimensional $\pi$-bonded chain of Si $p_{z}$ orbitals [68] and a large difference between the 


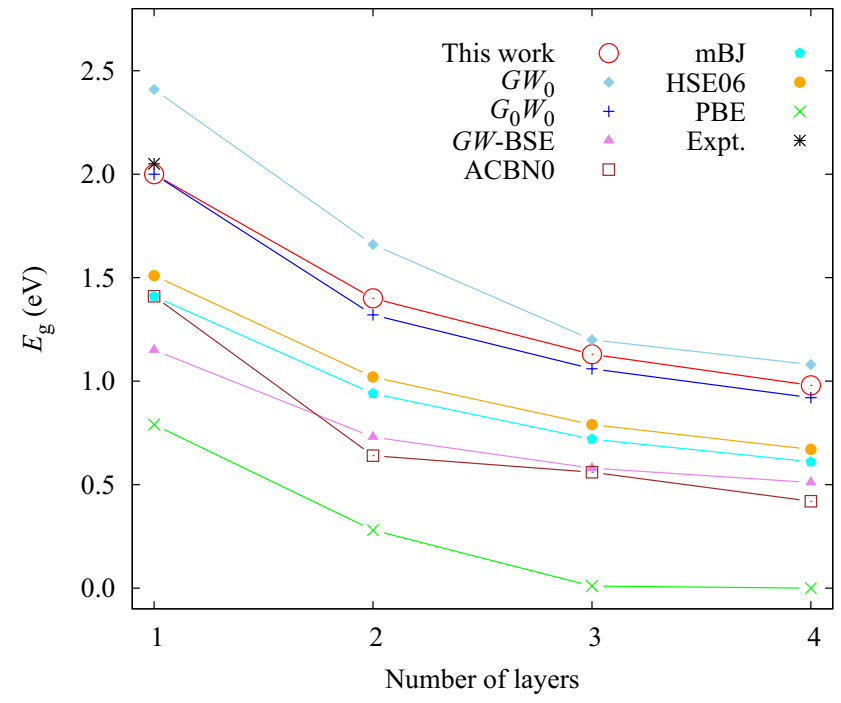

FIG. 3. Band gaps of black phosphorus as a function of a number of layers. We also list other gaps from PBE, HSE06 [65], mBJLDA [65], $G W_{0}$ [72], $G_{0} W_{0}$ [73], and $G W$-BSE [73]. An experimental band gap [74] is denoted with a black cross.

screenings on the surface and in bulk, it is a good test bed for a method to compute the surface and bulk gap simultaneously [64,69]. A 24-layer slab with $\sim 15 \AA$ vacuum was optimized with GGA until the residual forces on the atoms are less than $10^{-4} \mathrm{Ry} / \AA$. The kinetic energy cutoff is set to $80 \mathrm{Ry}$ and $d_{I J}$ to the nearest neighbors. The surface has two degenerate and coexisting reconstructions [70,71] as shown in Figs. 2(a) and 2(b) so that we compute the averaged surface band structures to compare with experiments. As shown in Fig. 2(c), the calculated averaged surface gap is $0.83 \mathrm{eV}$, agreeing well with the experimental value of $0.75 \mathrm{eV}[66,67]$, together with an accurate bulk gap. We note that the converged Hubbard parameters of $\mathrm{Si}$ atoms change spatially, reflecting the local variation of screening such that the calculated $U$ and $V$ are confirmed to gradually increase from the inside to the surface (not shown here).

Next, few-layer black phosphorus (BP) was chosen to test our method (Fig. 3). We used fully relaxed crystal structures using the revised van der Waals density functional (rev-vdWDF2) [75,76]. All intersite interactions between valence $s$ and $p$ electrons of the $\mathrm{P}$ atom within the plane are considered. Figure 3 shows the calculated band gaps in terms of the number of layers, together with other calculations and experiments. It is noticeable that, without including $V$, all ACBN0 gaps are quite smaller than the $G W$ gaps, and that HSE [65], mBJLDA [65], and ACBN0 produce the gaps close to the optical gaps by the $G W$-Bethe-Salpeter equation (BSE) method [73]. Considering the qualitative difference in shape of the optical spectrum between $G W$-BSE and HSE or other hybrid functionals $[64,77]$, we conclude that they underestimate the band gaps.

As shown in Fig. 3, our results are consistent with $G W$ results [72,73] and an available experiment [74]. We note that the computed band gaps of pure two-dimensional (2D) materials such as single-layer BP have a slight dependence on the range of the intersite Hubbard interaction (which will be

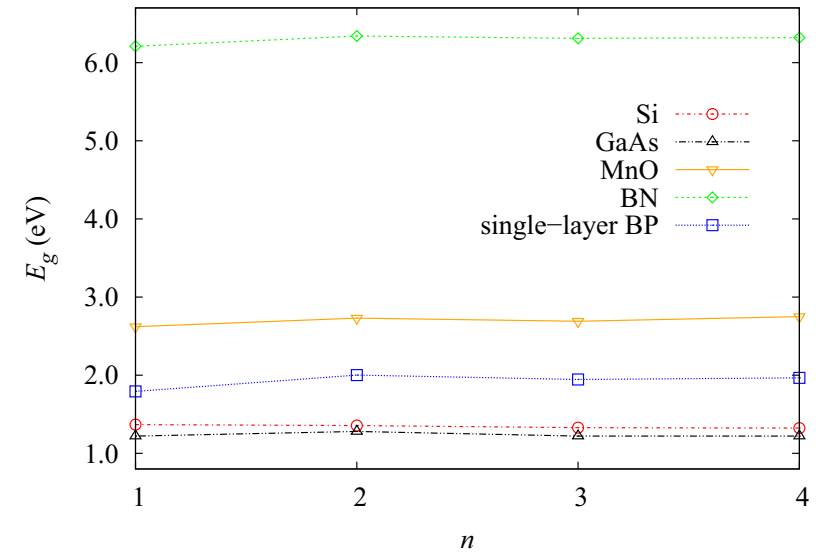

FIG. 4. Energy gap variations of $\mathrm{Si}, \mathrm{GaAs}, \mathrm{MnO}, \mathrm{BN}$, and a single-layer BP as a function of the distance between the pair atoms for the intersite Hubbard interaction. The abscissa denotes the $n$th nearest neighbor and the ordinate shows the gap with including $V$ 's up to the $n$th neighbor.

discussed in the next section), reflecting the complex nature of screening in low-dimensional materials [62]. Considering a large number of atoms in typical nanostructures, our method will have merit over the other computational methods that require quite expensive computational resources.

\section{DISCUSSION AND CONCLUSION}

The only empirical parameter in the present formalism is $d_{I J}$ that determines the range of a pair for the intersite $V$ in Eqs. (3) and (9). For the three-dimensional (3D) solids studied here, we find that the nearest-neighbor intersite interaction is enough to obtain the converged band-gap values. As shown in Fig. 4, the energy band gaps of 3D Si, GaAs, and MnO crystals show a negligible variation as increasing $d_{I J}$ while the zincblende structure $\mathrm{BN}$ shows a converged gap after including the second nearest neighbors. Unlike most 3D cases, the energy gaps for some low-dimensional systems show rather larger variations as a function of $d_{I J}$ than do 3D materials. For the case of the reconstructed $\operatorname{Si}(111)-(2 \times 1)$ surface discussed in the previous section, we do not need to include an intersite $V$ for the next-nearest-neighboring $\mathrm{Si}$ atoms. However, for a single-layer BP shown in Fig. 4, the gap with $V$ for the nearest neighbors is smaller (about 10\%) than one with $V$ for the next ones. Beyond this, the gap varies a little so that at least two sets of $V$ with different $d_{I J}$ are required to obtain a reasonably converged band gap of the single-layer BP. We note here that the increase in computational time with a longer $d_{I J}$ amounts to the increase in the DFT-GGA computation for a corresponding larger supercell case.

The role of self-consistent intersite Hubbard interactions on magnetic moments is also an interesting issue. For this, we calculated the magnetic moments of antiferromagnetic $\mathrm{MnO}$ and NiO. As shown in Table IV, the calculated magnetic moments are slightly reduced from the values using the ACBN0-PBE method [60] where the on-site $U$ enhances the localization of electrons at the atomic sites. On the other hand, the intersite $V$ reduces the on-site localization and shifts electrons to the bonding sites. Therefore, the competition between 
TABLE IV. Calculated magnetic moments in $\mu_{B}$ of antiferromangetic $\mathrm{MnO}$ and $\mathrm{NiO}$ and their comparisons with other works and experiments. Here, the moments are projected values for one spin orientation.

\begin{tabular}{lcc}
\hline \hline & $\mathrm{MnO}$ & $\mathrm{NiO}$ \\
\hline This work $(+V)$ & 4.69 & 1.78 \\
HSE [78] & 4.5 & 1.5 \\
ACBN0-PBE [60] & 4.79 & 1.83 \\
Experiments & $4.58[79], 4.79[80]$ & $1.77[80], 1.90[79,81]$ \\
\hline
\end{tabular}

$U$ and $V$ gives rise to the reduced magnetic moments compared with the ACBN0 method and the calculated moments are in excellent agreement with experiments [79-81].

In conclusion, we report an alternative $a b$ initio method for the electronic structures of solids employing a pseudohybrid density functional for extended Hubbard Coulomb interactions. We demonstrate that this method significantly improves the original ACBN0 functional in obtaining the band gaps of bulk and low-dimensional materials. Its self-consistent calculation can be done with a computational time comparable to DFT-GGA. With further validations with other methods $[82,83]$ and improvements of the current method such as noncollinear spin and forces [38], our method could fulfill requirements [6] for first-principles simulations suitable for massive database-driven materials research with an improved accuracy.

\section{ACKNOWLEDGMENTS}

We thank B.-H. Kim, H.-J. Kim, S.-H. Kang, S. Kim, S. Y. Park, S.-H. Jhi, H. J. Choi, and M. J. Han for fruitful discussions. Y.-W.S. was supported by NRF of Korea (Grant No. 2017R1A5A1014862, SRC program: vdWMRC center) and KIAS individual Grant No. (CG031509). Computations were supported by the CAC of KIAS. This work was also supported by the National Supercomputing Center with supercomputing resources including technical support (KSC2018-C2-0017).

\section{APPENDIX A: EFFECTS OF CROSS-EXCHANGE INTERACTIONS BETWEEN ORBITALS}

The mean-field (MF) expression of the electronic interaction energy in terms of atomic orbitals in its most general form can be written as

$$
E_{\mathrm{MF}}=\frac{1}{2} \sum_{I, J, K, L} \sum_{i j k l} \sum_{\sigma \sigma^{\prime}} E_{i j k l, \sigma \sigma^{\prime}}^{I J K L},
$$

where

$$
E_{i j k l, \sigma \sigma^{\prime}}^{I J K L}=\left\langle\phi_{i}^{I} \phi_{j}^{J}\left|V_{e e}\right| \phi_{k}^{K} \phi_{l}^{L}\right\rangle\left(n_{k i}^{K I \sigma} n_{l j}^{L J \sigma^{\prime}}-\delta_{\sigma \sigma^{\prime}} n_{k j}^{K J \sigma} n_{l i}^{L I \sigma^{\prime}}\right),
$$

where $n_{i j}^{I J \sigma}$ is defined in Eq. (2).

Considering $E_{i j k l, \sigma \sigma^{\prime}}^{I J K L}$, there are many possible arrangements for $I J K L$ and $i j k l$ [40], respectively. Among them, here we consider the first three large contributions, $E_{i j i j, \sigma \sigma^{\prime}}^{I I I I}$, $E_{i j i j, \sigma \sigma^{\prime}}^{I J J}$, and $E_{i j j i, \sigma \sigma^{\prime}}^{I J J I}$ where $I \neq J$. The first and second terms were discussed in the main text and correspond to on-site and intersite Hubbard interactions, respectively. The last one is the cross-charge exchanges between the neighboring atoms $I$ and
TABLE V. Calculated band gaps (in eV) with and without the cross-charge exchange in Eq. (A6). The " $+V$ " column summarizes

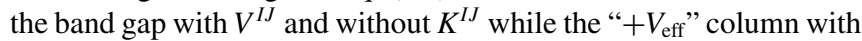
$V_{\text {eff }}^{I J}=V^{I J}-K^{I J}$. Experimental data for the energy gaps are from

\begin{tabular}{|c|c|c|c|c|c|}
\hline Solid & ACBN0 & $\begin{array}{l}\text { This work } \\
\quad(+V)\end{array}$ & $\begin{array}{l}\text { This work } \\
\left(+V_{\text {eff }}\right)\end{array}$ & $G W$ & Expt. \\
\hline C (A4) & 4.17 & 5.50 & 5.36 & 6.18 & 5.50 \\
\hline Si (A4) & 0.52 & 1.36 & 1.24 & 1.41 & 1.17 \\
\hline $\mathrm{Ge}(\mathrm{A} 4)$ & 0.00 & 0.61 & 0.48 & 0.95 & 0.74 \\
\hline BP (B3) & 1.24 & 2.27 & 2.14 & 2.20 & 2.40 \\
\hline AlP (B3) & 2.00 & 2.66 & 2.58 & 2.90 & 2.50 \\
\hline GaP (B3) & 1.74 & 2.47 & 2.39 & 2.80 & 2.35 \\
\hline InP (B3) & 0.94 & 1.46 & 1.41 & 1.44 & 1.42 \\
\hline AlAs (B3) & 1.75 & 2.43 & 2.35 & 2.18 & 2.23 \\
\hline GaAs (B3) & 0.68 & 1.28 & 1.21 & 1.85 & 1.52 \\
\hline InAs (B3) & 0.00 & 0.46 & 0.41 & 0.31 & 0.42 \\
\hline $\mathrm{SiC}$ (B3) & 1.74 & 2.49 & 2.39 & 2.88 & 2.42 \\
\hline BN (B3) & 5.14 & 6.31 & 6.17 & 7.14 & 6.36 \\
\hline ZnS (B3) & 3.43 & 3.71 & 3.68 & 4.15 & 3.80 \\
\hline $\mathrm{ZnSe}(\mathrm{B} 3)$ & 2.32 & 2.60 & 2.57 & 2.66 & 2.82 \\
\hline $\mathrm{ZnTe}(\mathrm{B} 3)$ & 1.99 & 2.30 & 2.28 & 2.15 & 2.39 \\
\hline LiF (B1) & 13.74 & 14.26 & 14.22 & 15.90 & 14.20 \\
\hline $\mathrm{MgO}(\mathrm{B} 1)$ & 8.84 & 10.06 & 9.93 & 9.16 & 7.90 \\
\hline $\mathrm{ZrO}_{2}$ & 5.10 & 5.97 & 5.95 & 5.34 & 5.50 \\
\hline $\mathrm{TiO}_{2}(\mathrm{C} 4)$ & 3.02 & 4.18 & 4.16 & 4.48 & 3.30 \\
\hline $\mathrm{MnO}(\mathrm{B} 1)$ & 2.56 & 2.73 & 2.71 & 3.50 & 3.60 \\
\hline $\mathrm{NiO}(\mathrm{B} 1)$ & 3.70 & 3.90 & 3.80 & 4.80 & 4.30 \\
\hline $\mathrm{ZnO}$ (B4) & 3.62 & 3.88 & 3.86 & 3.80 & 3.44 \\
\hline $\mathrm{Cu}_{2} \mathrm{O}(\mathrm{C} 3)$ & 1.28 & 1.52 & 1.50 & 1.97 & 2.17 \\
\hline MARE (\%) & 30.26 & 10.64 & 10.81 & 13.62 & \\
\hline MRE (\%) & -28.78 & 0.47 & -3.11 & 7.76 & \\
\hline
\end{tabular}
Refs. [28,29,37,47,84,85].

$\overline{\bar{a} \text { All data from self-consistent } G W(\operatorname{sc} G W) \text { calculation results in }}$ Ref. [25] except GaP, InP, AlAs from sc $G W$ in Ref. [53], ZnSe from $G_{0} W_{0}$ in Ref. [54], $\mathrm{ZrO}_{2}$ from $\mathrm{sc} G W$ in Ref. [56], $\mathrm{TiO}_{2}$ from $\mathrm{sc} G W$ in Ref. [57], and $\mathrm{Cu}_{2} \mathrm{O}$ from sc $G W$ in Ref. [58].

$J$ [40]. The first case where all $I J K L$ are equal will lead to the well-known Hubbard density functional for the LDA $+U$ method, and if we use a rotationally invariant on-site interaction $\left(E_{U}^{I}\right)$, Eq. (A1) will become the Dudarev $U$ functional [42]. The second term becomes the intersite Hubbard interaction as discussed in the main text.

Now, we consider the second and third ones together. If we use rotationally invariant forms for $\left\langle\phi_{i}^{I} \phi_{j}^{J}\left|V_{e e}\right| \phi_{k}^{K} \phi_{l}^{L}\right\rangle$ in Eq. (A2), we can rewrite the second interaction using $\left\langle\phi_{i}^{I} \phi_{j}^{J}\left|V_{e e}\right| \phi_{k}^{K} \phi_{l}^{L}\right\rangle=V^{I J} \delta_{I K} \delta_{J L} \delta_{i k} \delta_{j l}$ and $N^{I J} V^{I J}=\sum_{i, j}\left\langle\phi_{i}^{I} \phi_{j}^{J}\left|V_{e e}\right| \phi_{i}^{I} \phi_{j}^{J}\right\rangle$, where $N^{I J}$ is a number of degeneracy of the angular momentum for atoms $I$ and $J$ [40]. Likewise, for the third interactions, we can use $\left\langle\phi_{i}^{I} \phi_{j}^{J}\left|V_{e e}\right| \phi_{k}^{K} \phi_{l}^{L}\right\rangle=K^{I J} \delta_{I L} \delta_{J K} \delta_{i l} \delta_{j k} \quad$ and $N^{I J} K^{I J}=\sum_{i, j}\left\langle\phi_{i}^{I} \phi_{j}^{J}\left|V_{e e}\right| \phi_{j}^{J} \phi_{i}^{I}\right\rangle$. With these considerations, the MF energy in Eq. (A1) can be written as

$$
E_{\mathrm{Hub}}=\frac{1}{2}\left[\sum_{I} E_{U}^{I}+\sum_{\{I, J\}} E_{V}^{I J}+\sum_{\{I, J\}} E_{K}^{I J}\right],
$$

where the $\{I, J\}$ indicates the summation for a pair of atoms $I$ and $J$ within a given cutoff of $d_{I J}$. 
TABLE VI. Calculated band gaps (in eV) with two different sets of pseudopotentials. We tested the GBRV ultrasoft pseudopotentials [45] and the norm-conserving pseudopotentials provided by the PseudoDoJo project [46]. The two " $V$ " columns summarize the calculated band gaps with the current method. Experimental data for the energy gaps are from Refs. [28,29,37,47,84,85].

\begin{tabular}{|c|c|c|c|c|c|}
\hline Solid & $\mathrm{ACBNO}^{\mathrm{a}}$ & $\mathrm{ACBNO}^{\mathrm{b}}$ & $+V^{\mathrm{c}}$ & $+V^{\mathrm{d}}$ & Expt. \\
\hline C (A4) & 4.21 & 4.17 & 5.54 & 5.50 & 5.50 \\
\hline $\mathrm{Si}$ (A4) & 0.52 & 0.52 & 1.35 & 1.36 & 1.17 \\
\hline Ge (A4) & 0.00 & 0.00 & 0.60 & 0.61 & 0.74 \\
\hline BP (B3) & 1.24 & 1.24 & 2.27 & 2.27 & 2.40 \\
\hline AlP (B3) & 1.99 & 2.00 & 2.66 & 2.66 & 2.50 \\
\hline GaP (B3) & 1.78 & 1.74 & 2.47 & 2.47 & 2.35 \\
\hline InP (B3) & 1.00 & 0.94 & 1.46 & 1.46 & 1.42 \\
\hline AlAs (B3) & 1.76 & 1.75 & 2.43 & 2.43 & 2.23 \\
\hline GaAs (B3) & 0.70 & 0.68 & 1.22 & 1.28 & 1.52 \\
\hline InAs (B3) & 0.00 & 0.00 & 0.44 & 0.46 & 0.42 \\
\hline $\mathrm{SiC}$ (B3) & 1.73 & 1.74 & 2.49 & 2.49 & 2.42 \\
\hline BN (B3) & 5.21 & 5.14 & 6.39 & 6.31 & 6.36 \\
\hline ZnS (B3) & 4.30 & 3.43 & 4.77 & 3.71 & 3.80 \\
\hline ZnSe (B3) & 3.16 & 2.32 & 3.62 & 2.60 & 2.82 \\
\hline $\mathrm{ZnTe}$ (B3) & 2.73 & 1.99 & 3.28 & 2.30 & 2.39 \\
\hline $\mathrm{LiF}(\mathrm{B} 1)$ & 14.88 & 13.74 & 16.01 & 14.26 & 14.20 \\
\hline $\mathrm{MgO}(\mathrm{B} 1)$ & 8.89 & 8.84 & 10.16 & 10.06 & 7.90 \\
\hline $\mathrm{ZrO}_{2}$ & 5.32 & 5.10 & 6.19 & 5.97 & 5.50 \\
\hline $\mathrm{TiO}_{2}(\mathrm{C} 4)$ & 2.89 & 3.02 & 4.08 & 4.18 & 3.30 \\
\hline $\mathrm{MnO}(\mathrm{B} 1)$ & 2.88 & 2.56 & 3.55 & 2.73 & 3.60 \\
\hline $\mathrm{NiO}(\mathrm{B} 1)$ & 4.30 & 3.70 & 4.82 & 3.90 & 4.30 \\
\hline $\mathrm{ZnO}$ (B4) & 4.38 & 3.62 & 4.91 & 3.88 & 3.44 \\
\hline $\mathrm{Cu}_{2} \mathrm{O}(\mathrm{C} 3)$ & 1.63 & 1.28 & 2.02 & 1.52 & 2.17 \\
\hline MARE (\%) & 29.04 & 30.26 & 14.06 & 10.64 & \\
\hline MRE (\%) & -21.73 & -28.78 & 9.51 & 0.47 & \\
\hline
\end{tabular}

asseudoDoJo pseudopotential.

${ }^{\mathrm{b}}$ GBRV ultrasoft pseudopotential.

${ }^{c}$ PseudoDoJo pseudopotential.

${ }^{\mathrm{d}}$ GBRV ultrasoft pseudopotential.

Using the matrix notations of $\mathbf{n}^{I J}=\sum_{\sigma} \mathbf{n}^{I J \sigma}$ and $n^{I}=$ $\sum_{\sigma} \sum_{i} n_{i i}^{I I \sigma}$ for the general occupation in Eq. (2),

$$
\begin{aligned}
E_{V}^{I J}+E_{K}^{I J}= & V^{I J}\left[n^{I} n^{J}-\sum_{\sigma} \operatorname{Tr}\left[\mathbf{n}^{I J \sigma} \mathbf{n}^{J I \sigma}\right]\right] \\
& +K^{I J}\left[\operatorname{Tr}\left[\mathbf{n}^{I J} \mathbf{n}^{J I}\right]-\sum_{\sigma}\left[n^{I \sigma} n^{J \sigma}\right]\right] .
\end{aligned}
$$

We assume the fully localized limit for double counting as was also discussed in a previous work [40], so that the final expression for Hubbard pairwise energy is given by

$$
\begin{aligned}
E_{V}^{I J}+E_{K}^{I J}-E_{\mathrm{dc}} & =-V^{I J} \sum_{\sigma} \operatorname{Tr}\left[\mathbf{n}^{I J \sigma} \mathbf{n}^{J \sigma}\right]+K^{I J} \operatorname{Tr}\left[\mathbf{n}^{I J} \mathbf{n}^{J I}\right] \\
& \simeq-\left(V^{I J}-K^{I J}\right) \sum_{\sigma} \operatorname{Tr}\left[\mathbf{n}^{I J \sigma} \mathbf{n}^{J I \sigma}\right], \quad \text { (A5) }
\end{aligned}
$$

where we neglect $K^{I J} \sum_{\sigma \neq \sigma^{\prime}} \operatorname{Tr}\left[\mathbf{n}^{I J \sigma} \mathbf{n}^{J I \sigma^{\prime}}\right]$ owing to $V^{I J}-K^{I J} \gg K^{I J}$.

If we compare Eq. (A5) with the intersite Hubbard functional shown in Eq. (9) in the main text, we immediately notice that a replacement of $V^{I J}$ by $V_{\mathrm{eff}}^{I J}=V^{I J}-K^{I J}$ is enough for including the effects of cross-charge exchange between the pair of atoms $I$ and $J$.

Now, using Eqs. (4)-(6) in the main text, a pseudohybrid or ACBN0-like functional expression for $K^{I J}$ can be obtained in a straightforward way and the final expression can be written as

$$
K^{I J}=\frac{1}{2} \frac{\sum_{\sigma, \sigma^{\prime}} \sum_{i j k l}\left[\delta_{\sigma \sigma^{\prime}} P_{i k}^{I I \sigma} P_{j l}^{J J \sigma^{\prime}}-P_{i l}^{I J \sigma} P_{j k}^{J I \sigma^{\prime}}\right](i l \mid j k)}{\sum_{\sigma \sigma^{\prime}} \sum_{i j}\left[\delta_{\sigma \sigma^{\prime}} n_{i i}^{I I \sigma} n_{j j}^{J J \sigma^{\prime}}-n_{i j}^{I J \sigma} n_{j i}^{J I \sigma^{\prime}}\right]} .
$$

The effects of cross-exchange interactions on band gaps are summarized in Table S1. As shown in Table V, the calculated band gaps with and without the effects of $K$ are negligible. For the solids considered here, all computed gaps with $K$ are little bit smaller than the gap without $K$.

\section{APPENDIX B: EFFECTS OF CHOICE OF PSEUDOPOTENTIALS}

In this work, we used two kinds of pseudopotentials: PseudoDoJo norm-conserving [46] and GBRV ultrasoft [45] pseudopotentials. Table VI shows the effects of the choice of pseudopotentials. Almost all $s$ and $p$ electron systems considered here are not affected by the choice. However, particularly in the case of the $\mathrm{Zn}$ compound, the discrepancies are quite large.
[1] P. Hohenberg and W. Kohn, Inhomogeneous electron gas, Phys. Rev. 136, B864 (1964).

[2] W. Kohn and L. J. Sham, Self-consistent equations including exchange and correlation effects, Phys. Rev. 140, A1133 (1965).

[3] R. O. Jones, Density functional theory: Its origins, rise to prominence, and future, Rev. Mod. Phys. 87, 897 (2015).

[4] J. P. Perdew, K. Burke, and M. Ernzerhof, Generalized Gradient Approximation Made Simple, Phys. Rev. Lett. 77, 3865 (1996).

[5] S. Kümmel and L. Kronik, Orbital-dependent density functionals: Theory and applications, Rev. Mod. Phys. 80, 3 (2008).
[6] S. Curtarolo, G. L. W. Hart, M. Buongiorno Nardelli, N. Mingo, S. Sanvito, and O. Levy, The high-throughput highway to computational materials design, Nat. Mater. 12, 191 (2013).

[7] J. Greeley, T. F. Jaramillo, J. Bonde, I. Chorkendorff, and J. K. Nørskov, Computational high-throughput screening of electrocatalytic materials for hydrogen evolution, Nat. Mater. 5, 909 (2006).

[8] S. Wang, Z. Wang, W. Setyawan, N. Mingo, and S. Curtarolo, Assessing the Thermoelectric Properties of Sintered Compounds via High-Throughput $A b$-initio Calculations, Phys. Rev. X 1, 021012 (2011). 
[9] L. Yu and A. Zunger, Identification of Potential Photovoltaic Absorbers Based on First-Principles Spectroscopic Screening of Materials, Phys. Rev. Lett. 108, 068701 (2012).

[10] R. Armiento, B. Kozinsky, M. Fornari, and G. Ceder, Screening for high-performance piezoelectrics using highthroughput density functional theory, Phys. Rev. B 84, 014103 (2011).

[11] G. Hautier, A. Miglio, G. Ceder, G.-M. Rignanese, and X. Gonze, Identification and design principles of low hole effective mass $p$-type transparent conducting oxides, Nat. Commun. 4, 2292 (2013).

[12] K. Yim, Y. Yong, J. Lee, K. Lee, H.-H. Nahm, J. Yoo, C. Lee, C. Seong Hwang, and S. Han, Novel high- $\kappa$ dielectrics for nextgeneration electronic devices screened by automated $a b$ initio calculations, NPG Asia Mater. 7, e190 (2015).

[13] N. Mounet, M. Gibertini, P. Schwaller, D. Campi, A. Merkys, A. Marrazzo, T. Sohier, I. E. Castelli, A. Cepellotti, G. Pizzi, and N. Marzari, Two-dimensional materials from highthroughput computational exfoliation of experimentally known compounds, Nat. Nanotechnol. 13, 246 (2018).

[14] K. Yang, W. Setyawan, S. Wang, M. Buongiorno Nardelli, and S. Curtarolo, A search model for topological insulators with high-throughput robustness descriptors, Nat. Mater. 11, 614 (2012).

[15] T. Zhang, Y. Jiang, Z. Song, H. Huang, Y. He, Z. Fang, H. Weng, and C. Fang, Catalogue of topological electronic materials, Nature (London) 566, 475 (2019).

[16] M. G. Vergniory, L. Elcoro, C. Felser, N. Regnault, B. A. Bernevig, and Z. Wang, A complete catalogue of high-quality topological materials, Nature (London) 566, 480 (2019).

[17] F. Tang, H. C. Po, A. Vishwanath, and X. Wan, Comprehensive search for topological materials using symmetry indicators, Nature (London) 566, 486 (2019).

[18] V. I. Anisimov, J. Zaanen, and O. K. Andersen, Band theory and Mott insulators: Hubbard $U$ instead of Stoner I, Phys. Rev. B 44, 943 (1991).

[19] V. I. Anisimov, F. Aryasetiawan, and A. I. Lichtenstein, Firstprinciples calculations of the electronic structure and spectra of strongly correlated systems: The LDA $+U$ method, J. Phys.: Condens. Matter 9, 767 (1997).

[20] W. Metzner and D. Vollhardt, Correlated Lattice Fermions in $d=\infty$ Dimensions, Phys. Rev. Lett. 62, 324 (1989).

[21] A. Georges and G. Kotliar, Hubbard model in infinite dimensions, Phys. Rev. B 45, 6479 (1992).

[22] G. Kotliar, S. Y. Savrasov, K. Haule, V. S. Oudovenko, O. Parcollet, and C. A. Marianetti, Electronic structure calculations with dynamical mean-field theory, Rev. Mod. Phys. 78, 865 (2006).

[23] L. Hedin, New method for calculating the one-particle Green's function with application to the electron-gas problem, Phys. Rev. 139, A796 (1965).

[24] M. S. Hybertsen and S. G. Louie, Electron correlation in semiconductors and insulators: Band gaps and quasiparticle energies, Phys. Rev. B 34, 5390 (1986).

[25] M. Shishkin, M. Marsman, and G. Kresse, Accurate Quasiparticle Spectra from Self-Consistent $G W$ Calculations with Vertex Corrections, Phys. Rev. Lett. 99, 246403 (2007).

[26] J. Heyd, G. E. Scuseria, and M. Ernzerhof, Hybrid functionals based on a screened Coulomb potential, J. Chem. Phys. 118, 8207 (2003).
[27] B. G. Janesko, T. M. Henderson, and G. E. Scuseria, Screened hybrid density functionals for solid-state chemistry and physics, Phys. Chem. Chem. Phys. 11, 443 (2009).

[28] F. Tran and P. Blaha, Accurate Band Gaps of Semiconductors and Insulators with a Semilocal Exchange-Correlation Potential, Phys. Rev. Lett. 102, 226401 (2009).

[29] D. Koller, F. Tran, and P. Blaha, Merits and limits of the modified Becke-Johnson exchange potential, Phys. Rev. B 83, 195134 (2011).

[30] H. J. Kulik, M. Cococcioni, D. A. Scherlis, and N. Marzari, Density Functional Theory in Transition-Metal Chemistry: A Self-Consistent Hubbard $U$ Approach, Phys. Rev. Lett. 97, 103001 (2006).

[31] M. Cococcioni and S. de Gironcoli, Linear response approach to the calculation of the effective interaction parameters in the LDA $+U$ method, Phys. Rev. B 71, 035105 (2005).

[32] T. Miyake and F. Aryasetiawan, Screened Coulomb interaction in the maximally localized Wannier basis, Phys. Rev. B 77, 085122 (2008).

[33] M. Aichhorn, L. Pourovskii, V. Vildosola, M. Ferrero, O. Parcollet, T. Miyake, A. Georges, and S. Biermann, Dynamical mean-field theory within an augmented plane-wave framework: Assessing electronic correlations in the iron pnictide $\mathrm{LaFeAsO}$, Phys. Rev. B 80, 085101 (2009).

[34] T. Miyake, F. Aryasetiawan, and M. Imada, Ab initio procedure for constructing effective models of correlated materials with entangled band structure, Phys. Rev. B 80, 155134 (2009).

[35] N. J. Mosey and E. A. Carter, Ab initio evaluation of Coulomb and exchange parameters for DFT $+U$ calculations, Phys. Rev. B 76, 155123 (2007).

[36] N. J. Mosey, P. Liao, and E. A. Carter, Rotationally invariant $a b$ initio evaluation of Coulomb and exchange parameters for DFT $+U$ calculations, J. Chem. Phys. 129, 014103 (2008).

[37] L. A. Agapito, S. Curtarolo, and M. Buongiorno Nardelli, Reformulation of DFT $+U$ as a Pseudohybrid Hubbard Density Functional for Accelerated Materials Discovery, Phys. Rev. X 5, 011006 (2015).

[38] N. Tancogne-Dejean, M. J. T. Oliveira, and A. Rubio, Selfconsistent DFT $+U$ method for real-space time-dependent density functional theory calculations, Phys. Rev. B 96, 245133 (2017).

[39] V. I. Anisimov, I. S. Elfimov, N. Hamada, and K. Terakura, Charge-ordered insulating state of $\mathrm{Fe}_{3} \mathrm{O}_{4}$ from first-principles electronic structure calculations, Phys. Rev. B 54, 4387 (1996).

[40] V. L. Campo, Jr., and M. Cococcioni, Extended DFT $+U+$ $V$ method with on-site and inter-site electronic interactions, J. Phys.: Condens. Matter 22, 055602 (2010).

[41] H. J. Kulik and N. Marzari, Transition-metal dioxides: A case for the intersite term in Hubbard-model functionals, J. Chem. Phys. 134, 094103 (2011).

[42] S. L. Dudarev, G. A. Botton, S. Y. Savrasov, C. J. Humphreys, and A. P. Sutton, Electron-energy-loss spectra and the structural stability of nickel oxide: An LSDA $+U$ study, Phys. Rev. B 57, 1505 (1998).

[43] P. Giannozzi, S. Baroni, N. Bonini, M. Calandra, R. Car, C. Cavazzoni, D. Ceresoli, G. L. Chiarotti, M. Cococcioni, I. Dabo, A. D. Corso, S. de Gironcoli, S. Fabris, G. Fratesi, R. Gebauer, U. Gerstmann, C. Gougoussis, A. Kokalj, M. Lazzeri, L. Martin-Samos et al., QUANTUM ESPRESSO: A modular 
and open-source software project for quantum simulations of materials, J. Phys.: Condens. Matter 21, 395502 (2009).

[44] R. P. Muller, PyQuante 1.6.4: Python Quantum Chemistry, http://pyquante.sourceforge.net/.

[45] K. F. Garrity, J. W. Bennett, K. M. Rabe, and D. Vanderbilt, Pseudopotentials for high-throughput DFT calculations, Comput. Mater. 81, 446 (2014).

[46] M. van Setten, M. Giantomassi, E. Bousquet, M. Verstraete, D. Hamann, X. Gonze, and G.-M. Rignanese, The pseudodojo: Training and grading a 85 element optimized norm-conserving pseudopotential table, Comput. Phys. Commun. 226, 39 (2018).

[47] M. J. Lucero, T. M. Henderson, and G. E. Scuseria, Improved semiconductor lattice parameters and band gaps from a middlerange screened hybrid exchange functional, J. Phys.: Condens. Matter 24, 145504 (2012).

[48] A. J. Garza and G. E. Scuseria, Predicting band gaps with hybrid density functionals, J. Phys. Chem. Lett. 7, 4165 (2016).

[49] J.-H. Yuan, Q. Chen, L. R. C. Fonseca, M. Xu, K.-H. Xue, and X.-S. Miao, GGA-1/2 self-energy correction for accurate band structure calculations: The case of resistive switching oxides, J. Phys. Commun. 2, 105005 (2018).

[50] H. Nakai, J. Heyd, and G. E. Scuseria, Periodic-boundarycondition calculation using Heyd-Scuseria-Ernzerhof screened Coulomb hybrid functional: Electronic structure of anatase and rutile $\mathrm{TiO}_{2}$, J. Comput. Chem. Jpn. 5, 7 (2006).

[51] F. Fuchs, J. Furthmüller, F. Bechstedt, M. Shishkin, and G. Kresse, Quasiparticle band structure based on a generalized Kohn-Sham scheme, Phys. Rev. B 76, 115109 (2007).

[52] M. Heinemann, B. Eifert, and C. Heiliger, Band structure and phase stability of the copper oxides $\mathrm{Cu}_{2} \mathrm{O}, \mathrm{CuO}$, and $\mathrm{Cu}_{4} \mathrm{O}_{3}$, Phys. Rev. B 87, 115111 (2013).

[53] I. N. Remediakis and E. Kaxiras, Band-structure calculations for semiconductors within generalized-density-functional theory, Phys. Rev. B 59, 5536 (1999).

[54] Y. Hinuma, A. Grüneis, G. Kresse, and F. Oba, Band alignment of semiconductors from density-functional theory and manybody perturbation theory, Phys. Rev. B 90, 155405 (2014).

[55] H. Jiang and P. Blaha, $G W$ with linearized augmented plane waves extended by high-energy local orbitals, Phys. Rev. B 93, 115203 (2016).

[56] H. Jiang, R. I. Gomez-Abal, P. Rinke, and M. Scheffler, Electronic band structure of zirconia and hafnia polymorphs from the $G W$ perspective, Phys. Rev. B 81, 085119 (2010).

[57] S. Lany, Band-structure calculations for the $3 d$ transition metal oxides in GW, Phys. Rev. B 87, 085112 (2013).

[58] F. Bruneval, N. Vast, L. Reining, M. Izquierdo, F. Sirotti, and N. Barrett, Exchange and Correlation Effects in Electronic Excitations of $\mathrm{Cu}_{2} \mathrm{O}$, Phys. Rev. Lett. 97, 267601 (2006).

[59] See Supplemental Material at http://link.aps.org/supplemental/ 10.1103/PhysRevResearch.2.043410 for effects of additional exchange terms, pseudopotential dependence, and comparisons of calculated band structures.

[60] P. Gopal, R. D. Gennaro, M. S. dos Santos Gusmao, R. A. R. A. Orabi, H. Wang, S. Curtarolo, M. Fornari, and M. Buongiorno Nardelli, Improved electronic structure and magnetic exchange interactions in transition metal oxides, J. Phys.: Condens. Matter 29, 444003 (2017).
[61] H. J. Kulik and N. Marzari, A self-consistent Hubbard $U$ density-functional theory approach to the addition-elimination reactions of hydrocarbons on bare $\mathrm{FeO}^{+}$, J. Chem. Phys. 129, 134314 (2008).

[62] D. Y. Qiu, F. H. da Jornada, and S. G. Louie, Screening and many-body effects in two-dimensional crystals: Monolayer $\mathrm{MoS}_{2}$, Phys. Rev. B 93, 235435 (2016).

[63] F. A. Rasmussen, P. S. Schmidt, K. T. Winther, and K. S. Thygesen, Efficient many-body calculations for twodimensional materials using exact limits for the screened potential: Band gaps of $\mathrm{MoS}_{2}, h-\mathrm{BN}$, and phosphorene, Phys. Rev. B 94, 155406 (2016).

[64] M. Jain, J. R. Chelikowsky, and S. G. Louie, Reliability of Hybrid Functionals in Predicting Band Gaps, Phys. Rev. Lett. 107, 216806 (2011).

[65] J. Qiao, X. Kong, Z.-X. Hu, F. Yang, and W. Ji, High-mobility transport anisotropy and linear dichroism in few-layer black phosphorus, Nat. Commun. 5, 4475 (2014).

[66] R. I. G. Uhrberg, G. V. Hansson, J. M. Nicholls, and S. A. Flodström, Experimental Evidence for One Highly Dispersive Dangling-Bond Band on Si(111)-2 × 1, Phys. Rev. Lett. 48, 1032 (1982).

[67] P. Perfetti, J. M. Nicholls, and B. Reihl, Unoccupied surface-state band on Si(111)-2 × 1, Phys. Rev. B 36, 6160 (1987).

[68] K. C. Pandey, New $\pi$-Bonded Chain Model for Si(111)- $2 \times 1$ Surface, Phys. Rev. Lett. 47, 1913 (1981).

[69] M. Rohlfing and S. G. Louie, Excitons and Optical Spectrum of the Si(111)- $2 \times 1$ Surface, Phys. Rev. Lett. 83, 856 (1999).

[70] K. Löser, M. Wenderoth, T. K. A. Spaeth, J. K. Garleff, R. G. Ulbrich, M. Pötter, and M. Rohlfing, Spectroscopy of positively and negatively buckled domains on $\operatorname{Si}(111)-2 \times 1$, Phys. Rev. B 86, 085303 (2012).

[71] C. Violante, L. Chiodo, A. M. Conte, F. Bechstedt, and O. Pulci, $\operatorname{Si}(111) 2 \times 1$ surface isomers: DFT investigations on stability and doping effects, Surf. Sci. 621, 123 (2014).

[72] V. Wang, Y. Kawazoe, and W. T. Geng, Native point defects in few-layer phosphorene, Phys. Rev. B 91, 045433 (2015).

[73] A. N. Rudenko, S. Yuan, and M. I. Katsnelson, Toward a realistic description of multilayer black phosphorus: From $G W$ approximation to large-scale tight-binding simulations, Phys. Rev. B 92, 085419 (2015).

[74] L. Liang, W. Lin, B. G. Sumpter, V. Meunier, and M. Pan, Electronic bandgap and edge reconstruction in phosphorene materials, Nano Lett. 14, 6400 (2014).

[75] I. Hamada, van der Waals density functional made accurate, Phys. Rev. B 89, 121103(R) (2014).

[76] H.-J. Kim, S.-H. Kang, I. Hamada, and Y.-W. Son, Origins of the structural phase transitions in $\mathrm{MoTe}_{2}$ and $\mathrm{WTe}_{2}$, Phys. Rev. B 95, 180101(R) (2017).

[77] V. Tran, R. Soklaski, Y. Liang, and L. Yang, Layercontrolled band gap and anisotropic excitons in few-layer black phosphorus, Phys. Rev. B 89, 235319 (2014).

[78] C. Rödl, F. Fuchs, J. Furthmüller, and F. Bechstedt, Quasiparticle band structures of the antiferromagnetic transition-metal 
oxides $\mathrm{MnO}, \mathrm{FeO}, \mathrm{CoO}$, and $\mathrm{NiO}$, Phys. Rev. B 79, 235114 (2009).

[79] A. K. Cheetham and D. A. O. Hope, Magnetic ordering and exchange effects in the antiferromagnetic solid solutions $\mathrm{Mn}_{x} \mathrm{Ni}_{1-x} \mathrm{O}$, Phys. Rev. B 27, 6964 (1983).

[80] B. E. F. Fender, A. J. Jacobson, and F. A. Wedgwood, Covalency parameters in $\mathrm{MnO}, \alpha-\mathrm{MnS}$, and $\mathrm{NiO}$, J. Chem. Phys. 48, 990 (1968).

[81] W. L. Roth, Magnetic structures of $\mathrm{MnO}, \mathrm{FeO}, \mathrm{CoO}$, and $\mathrm{NiO}$, Phys. Rev. 110, 1333 (1958).

[82] J. Huang, S.-H. Lee, Y.-W. Son, A. Supka, and S. Liu, First-principles study of two-dimensional ferroelectrics using self-consistent Hubbard parameters, Phys. Rev. B 102, 165157 (2020).

[83] I. Timrov, N. Marzari, and M. Cococcioni, Self-consistent Hubbard parameters from density-functional perturbation theory in the ultrasoft and projector-augmented wave formulations, arXiv:2011.03271.

[84] Y. Tezuka, S. Shin, T. Ishii, T. Ejima, S. Suzuki, and S. Sato, Photoemission and bremsstrahlung isochromat spectroscopy studies of $\mathrm{TiO}_{2}$ (rutile) and $\mathrm{SrTiO}_{3}$, J. Phys. Soc. Jpn. 63, 347 (1994).

[85] G. A. Sawatzky and J. W. Allen, Magnitude and Origin of the Band Gap in NiO, Phys. Rev. Lett. 53, 2339 (1984). 